\title{
Kidney cancer survivorship survey of urologists and survivors: The gap in perceptions of care, but agreement on needs
}

\author{
Patricia Moretto, MD; Michael A.S. Jewett, MD, FRCSC; ${ }^{\dagger}$ Joan Basiuk, RN; ${ }^{\xi}$ Deborah Maskens, ${ }^{*}$ \\ Christina M. Canil, $M D^{*}$
}

*Division of Medical Oncology, Department of Medicine, The Ottawa Hospital Cancer Centre, The Ottawa Research Institute and University of Ottawa, Ottawa ON; 'Division of Urology, Departments of Surgery and Surgical Oncology, University of Toronto, Princess Margaret Cancer Centre, University Health Network, Toronto ON; sKidney Cancer Research Network of Canada, Princess Margaret Cancer Centre, Toronto ON; * Kidney Cancer Canada

Cite as: Can Urol Assoc J 2014;8(5-6):190-4. http://dx.doi.org/10.5489/cuaj.1907

Published online June 16, 2014.

\section{Abstract}

Introduction: There is lack of evidence-based literature addressing comprehensive long-term care for kidney cancer (KC) survivors. Additionally, it is unclear if the concerns of KC patients/caregivers are being adequately addressed. Therefore, Kidney Cancer Canada, a patient-led support organization for Canadians with $\mathrm{KC}$, commissioned this first recorded survivorship survey specific to $\mathrm{KC}$ patients/caregivers.

Methods: We conducted a cross-sectional online survey of Canadian patients/caregivers diagnosed with localized $\mathrm{KC}$, and a separate parallel survey of Canadian urologists. The primary objectives were to assess patient/caregivers' and urologists' perceptions of information provided, as well as the physical/psychological/ emotional impact of $\mathrm{KC}$ treatment.

Results: Urologists recalled providing information about surgical complications (90\%) and their management (63\%), while patients/ caregiver recalled much less $(33 \%$ and $35 \%)$. Of the urologists, $93 \%$ recalled providing information on cancer recurrence, but only $42 \%$ of patients/caregivers remembered receiving this information. Concerns identified by patients/caregivers and urologists were similar: fear of recurrence, concerns about cancer, fatigue, and anxiety. Importantly, all agreed that survivorship information was paramount. Education of both patients/caregivers and physicians and the development of guidelines were factors identified to ensure optimal KC survivorship. Study limitations include potential biases in recall and selection of participants.

Conclusion: There was some discordance between urologists' and patients/caregivers' rates of recall of information provided. Patients/ caregivers would have desired more information about their cancer, long-term follow-up, and potential complications. A survivorship care plan (SCP) tailored to KC may be an effective measure to address these needs. The impact of this SCP on survivor outcomes should be rigorously assessed.

\section{Introduction}

With diagnostic and treatment advances, kidney cancer (KC) is being diagnosed more frequently and at an earlier stage with improved outcomes. ${ }^{1}$ With newer enhanced systemic therapies, patients with advanced disease are living longer. ${ }^{2}$ These combined factors have resulted in an increased number of KC survivors. Recent Surveillance Epidemiology and End Results (SEER) data show that 1 in 5 adults over the age of 65 is a cancer survivor. ${ }^{3}$ The definition of a cancer survivor may vary, but the National Cancer Institute uses the following definition: "an individual is a cancer survivor from the time of diagnosis to the balance of his or her life. Family members, friends, and caregivers are also impacted and included in this definition." ${ }^{4}$

In 2005, the Institute of Medicine (IOM) published the landmark report From Cancer Patient to Cancer Survivor: Lost in Transition. ${ }^{5}$ This widely cited publication defined the needs involved in survivorship care (prevention and detection of new cancers, surveillance for cancer recurrence or new cancers, long term toxicity management, psychological distress management, and coordination of care between specialists and primary care providers). However, to date, there has been little research in survivorship care for KC patients. In fact, based on the September 2011 report by the Canadian Cancer Research Alliance, KC was identified as one of only two tumour sites for which no money was spent to support survivorship research. ${ }^{6}$

Kidney Cancer Canada (KCC), a patient-led support organization for Canadians diagnosed with kidney cancer, commissioned the first recorded survivorship survey specific to patients and their caregivers diagnosed with localized Stage I-III KC. ${ }^{7}$ The aims of the survey were: (1) to assess perceptions of KC patients/caregivers and urologists regarding the information received/provided about $\mathrm{KC}$ before and after surgery (partial or radical nephrectomy); (2) to assess how these perceptions may affect KC survivorship care; and (3) to identify ways of improving KC survivorship. 


\section{Methods}

A cross-sectional survey of Canadian patients diagnosed with non-metastatic KC and their caregivers, and a separate parallel survey for Canadian urologists, were developed by KCC with the assistance of a research marketing firm, Leger (www.leger360.com). Content was also reviewed by a uro-oncologist with $\mathrm{KC}$ expertise (MJ). Arms-length funding was provided by Pfizer Canada. Surveys were electronically distributed. The target population was: (1) non-metastatic (at diagnosis) KC patients and their caregivers and (2) urologists who treat $\mathrm{KC}$. The sampling frame consisted of nonmetastatic (at diagnosis) KC patients and their caregivers whose e-mail addresses were available to KCC. In addition, a survey link was also posted on the KCC website and social media was used through Facebook and Twitter. Urologists were recruited from a medical professional panel available to Leger and contacted via e-mail.

Patients/caregivers and urologists received 1 e-mail invitation only, which included the study rationale and instructions on how to access the online survey. Completion of the survey signified informed consent. Participation was voluntary. Physicians were offered a $\$ 100$ honorarium upon completion of the study. Results were anonymized and had no direct impact on patients' care. The survey was available in English and French.

The patient/caregiver survey consisted of 35 questions, while the urologist survey consisted of 22 questions. Both contained similar questions regarding demographics, $\mathrm{KC}$ diagnosis, treatment, follow-up care and expectations. Question format was multiple choice and multi-point rating, and participants chose the best available answer.

The primary objective of this study was to assess whether there was a difference between patients/caregivers and urologists' perceptions on information given, and on the physical, psychological/emotional impact of KC treatment. Descriptive analysis was performed using Microsoft Excel.

\section{Results}

A total of 361 surveys were completed: 276 by patients, 45 by caregivers, and 40 by urologists. The Northwest Territories, Yukon, and Nunavut were not represented in this sample, as there were no KCC members from those areas at the time of the study. A total of 161 urologists were invited to participate in the study, with a response rate of $25 \%$. The response rate for patients/caregivers was not possible to determine as social media was used and we do not know how many non-KCC members looked at the survey and chose not to participate. However, the KCC patient/caregiver membership with email addresses was 979 during the survey period. A total of 558 surveys were initiated, but 237 were excluded due to initial diagnosis of metastatic disease, residence outside of Canada, age under 18, or never been diagnosed with kidney cancer/or never cared for someone with kidney cancer. The remaining 321 completed surveys were used for analysis. Most of the patient/caregiver respondents were in Ontario ( $42 \%$ and $56 \%$, respectively), followed by Alberta $(11 \%$ and $16 \%)$, Quebec (20\% and 7\%), and British Columbia (12\% and $9 \%)$. Patient respondents were mostly male (56\%), but most of the caregiver respondents $(84 \%)$ were females. The most frequent age range for both patients and caregivers was 55 to 64 years (31\% and 38\%, respectively). Of 276 patient respondents, all but 4 underwent nephrectomy or partial nephrectomy for KC. Of the patient respondents, $57 \%$ had been initially diagnosed with stage I or II disease and $62 \%$ were diagnosed with kidney cancer within the past 3 years. When questioned about their current status of disease, $69 \%$ of patients reported "no evidence of disease."

Most respondent urologists were from Ontario (43\%), followed by British Columbia (25\%), Quebec $(8 \%)$ and New Brunswick (8\%), with $70 \%$ having treated $\mathrm{KC}$ for more than 10 years.

In this survey, urologists, patients and caregivers were asked to recall what information, regarding their kidney cancer, was provided before and after surgery. Ninety-three percent $(93 \%)$ of urologists proactively provided patients detailed information about their cancer before surgery. On the other hand, $51 \%$ of those affected by KC (patients and caregivers) "wished their urologists had given more information" about the KC prior to surgery. Nearly all the urologists $(98 \%)$ proactively provided patients detailed information about their cancer following surgery. However, 69\% of patients/caregivers thought they had received clear information, but $62 \%$ "wished they had been given more information" regarding their kidney cancer after surgery. Most urologists and patients/caregivers reported having a discussion regarding the results of the surgery, however, the recall regarding the specific details of the $\mathrm{KC}$, such as tumour stage, type, grade/aggressiveness and lymph node involvement, was different between both groups (Fig. 1).

Urologists recalled providing information about surgical complications and their management $(90 \%$ and $63 \%$, respectively), including late-term health outcomes, such as hypertension and renal dysfunction. The patients/caregivers recall on those matters was $33 \%$ and $35 \%$, respectively. Most (93\%) of urologist recalled providing information about recurrence risks, but only $42 \%$ of patients/caregivers remembered receiving this information.

On assessment of the physical and psychological/ emotional impact of $\mathrm{KC}, 87 \%$ of patients/caregivers were affected in at least one of these domains, while urologists estimated that about $30 \%$ of patients/caregivers would be affected. Figure 2 highlights the proportion of impact on specific details for each domain as estimated by respondent urologists and as stated by patients/caregivers. Despite the 
Moretto et al.

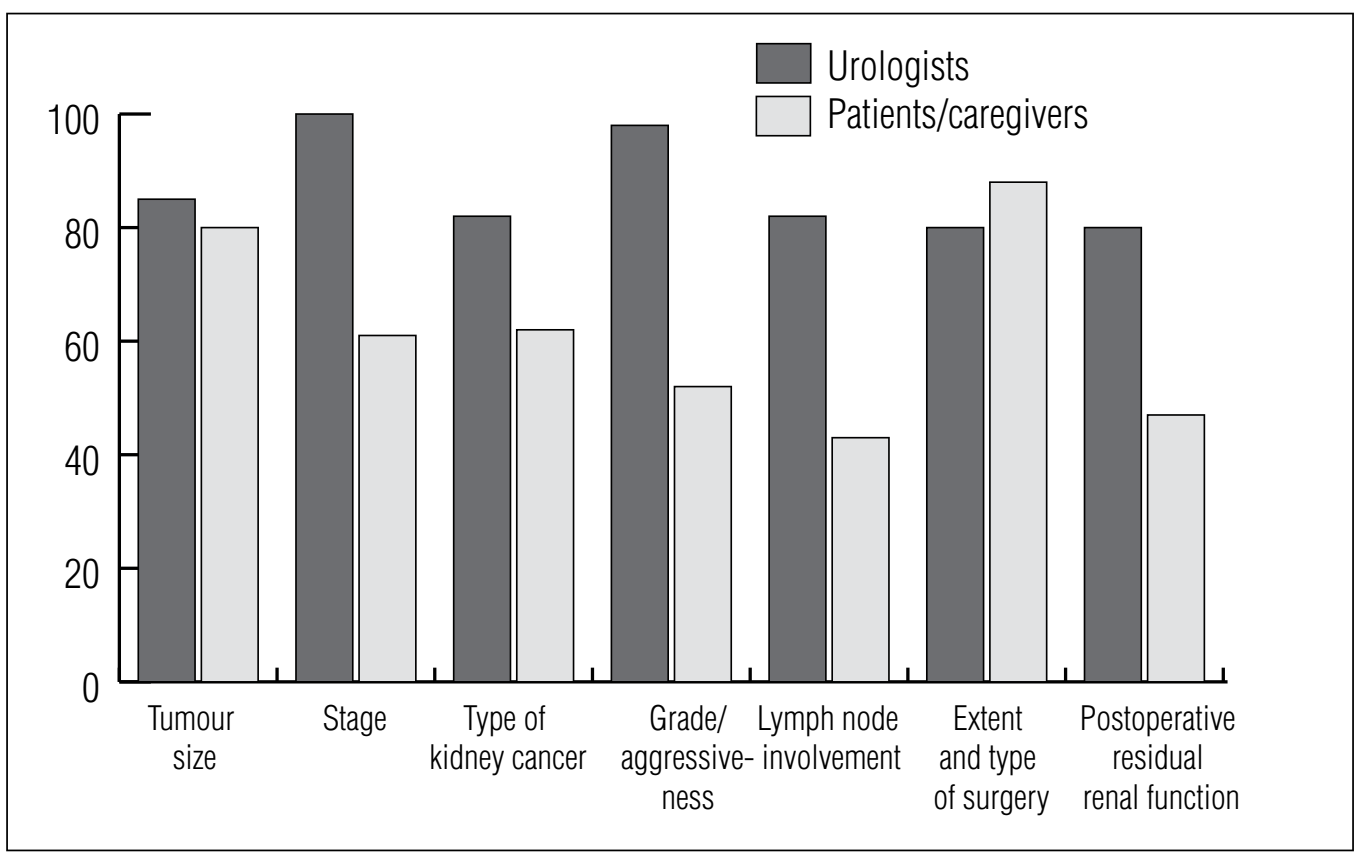

Fig. 1. Percentage of urologist vs. patients/caregiver recall of specific details, provided after surgery, of their kidney cancer, surgery and postoperative residual renal function.

potential physical and psychological/emotional impact of cancer surgery, $78 \%$ of urologists thought their patients were prepared to recognize and deal with these issues; in reality, only $48 \%$ of those affected by KC felt prepared.

The most commonly prescribed instructions given to patients/caregivers as recalled by urologists were: maintain adequate blood pressure (BP) control (88\%), quit smoking $(88 \%)$ and follow a healthy lifestyle $(83 \%)$. As recalled by patients/caregivers, the most common advice given by urologists were: see a family doctor annually ( $40 \%$ and $40 \%$ ), follow a healthy lifestyle (39\% and $37 \%$ ), drink plenty of fluids $(37 \%$ and $28 \%$ ), and maintain adequate BP control (31\% and 39\%).

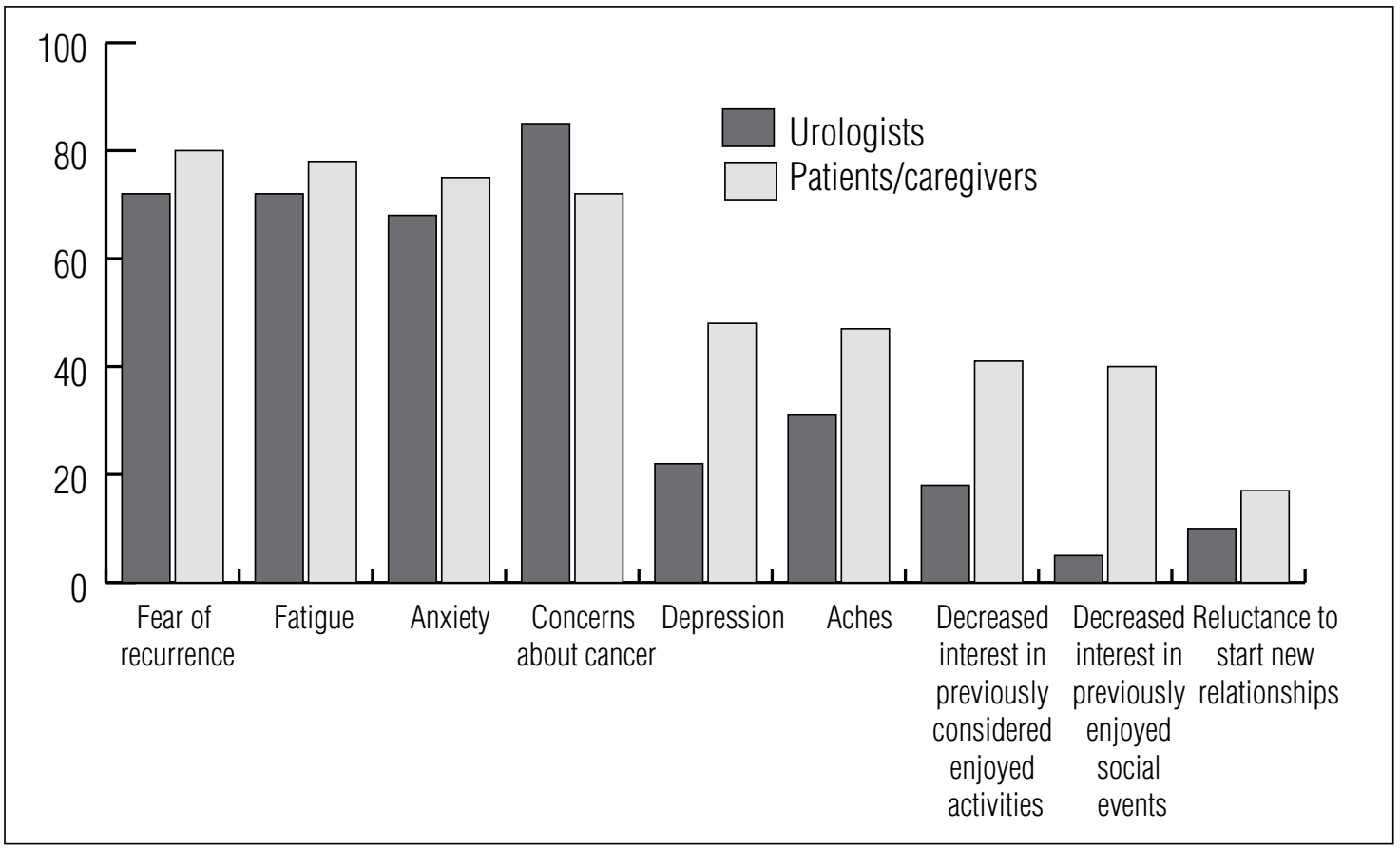

Fig. 2. Percentages of the most frequently identified physical and psychological/emotional concerns by urologist versus patient/ caregivers. 
While virtually all patients/caregivers and urologists agreed that survivorship information is important, patients/ caregivers placed greater importance on this than urologists (91\% vs. $68 \%$ respectively).

There was consensus among urologists and patients/ caregivers for the need of a survivorship care plan (SCP). According to the urologists' surveyed, the most frequent barriers to the implementation of a SCP were lack of resources $(78 \%)$, standards of care for survivorship (68\%), physician education and training (63\%), and patient education (60\%). Similarly, both groups agreed on identified actions to ensure optimal long-term survivorship (Table 1).

\section{Discussion}

The cancer journey continues after the completion of treatment. Patients and their caregivers are left with physical, psychological, and emotional sequelae. It is becoming increasing clear that many of the patient/caregiver concerns have not been fully addressed. ${ }^{8}$

There are no comprehensive guidelines for the proper long-term care for KC survivors as advised by the IOM. ${ }^{9}$ This is due, in part, to the absence of evidence-based literature to guide the development of a guideline. ${ }^{10}$ The Canadian Urological Association (CUA) has published follow-up guidelines with suggested imaging, physical examination, and blood work for patients' post-nephrectomy based on tumour stage to assess possible recurrence. ${ }^{11}$ However, it does not address potential long-term complications.

Our survey identified important domains in survivorship care including details of cancer diagnosis and treatment, toxicities/complications of therapy, risk of recurrence, lifestyle modifications and identification of psychosocial needs and resources. Our survey results showed that patients/caregivers did not recall having these issues addressed, while urologists were under the impression the issues were addressed in some capacity. Possible explanations for this discrepancy may include: a decrease in retention of information by patients/caregivers due to the overwhelming ordeal of being faced with a cancer diagnosis, differing emphasis on the importance given to specific issues between doctors and patients/caregivers, or lack of information in a written format. Time constraints and limited resources for physicians to provide comprehensive survivorship care also act as an important barrier. ${ }^{10}$ Providing patients/caregivers with an electronic or written document outlining the survivorship plan would act as a reminder and resource and also ensure that physicians do not omit or "gloss over" important issues.

Our survey indicates that KC patients/caregivers and urologists agree that a medically endorsed survivorship website would be useful. Ideally, this website would allow healthcare professionals, patients, or caregivers to input specific details, such as diagnosis (pathology), details of surgery,

\begin{tabular}{|c|c|c|}
\hline & $\begin{array}{l}\text { Patients/ } \\
\text { caregivers }\end{array}$ & Urologist \\
\hline $\begin{array}{l}\text { Provide family physicians with more } \\
\text { information on the specific needs of KC } \\
\text { survivors }\end{array}$ & $72 \%$ & $75 \%$ \\
\hline $\begin{array}{l}\text { Develop national guidelines for long- } \\
\text { term follow-up care and monitoring of } \\
\text { KC survivors }\end{array}$ & $75 \%$ & $85 \%$ \\
\hline $\begin{array}{l}\text { Provide patients and caregivers with } \\
\text { more formalized survivorship care } \\
\text { information and plans }\end{array}$ & $75 \%$ & $65 \%$ \\
\hline $\begin{array}{l}\text { Educate healthcare providers on the } \\
\text { importance of providing long-term } \\
\text { survivorship care information to KC } \\
\text { patients }\end{array}$ & $76 \%$ & $70 \%$ \\
\hline $\begin{array}{l}\text { Educate } \mathrm{KC} \text { patients on the importance } \\
\text { of following a long-term survivorship } \\
\text { plan }\end{array}$ & $81 \%$ & $83 \%$ \\
\hline
\end{tabular}

and physician contact information. These data would then be part of an electronic individualized SCP that would also include appropriate follow-up schedule, late toxicities and lifestyle modifications, and community resources.

The optimal design of a survivorship program for $\mathrm{KC}$ is unclear. A phase III trial of survivorship in breast cancer should be noted. Patients were randomized to receive an extensive SCP versus "a standard discharge visit with the oncologist and a discharge letter to the primary care physician." There was no difference in satisfaction/quality of life, care coordination or use of health services between the extensive SCP and the standard discharge visit and letter. ${ }^{12}$

As per the American Society of Clinical Oncology (ASCO) recommendations to improve survivor care, ${ }^{12}$ we should educate and empower cancer survivors and caregivers to advocate for their unique needs and ensure optimal longterm health. The simple act of offering educational information, as with the KCC website, and participation in this survey already raise awareness and help achieve these goals. However, based on the results of our survey, it is clear that the $\mathrm{KC}$ patients and caregivers desire more information.

There are several limitations to our study. Due to the nature of this survey, information on response rates could not be captured for the patient/caregiver group. In addition, there is potential selection bias related to computer access and literacy, as KC patients/caregivers must have had an e-mail address registered with KCC or internet access to social media to participate. The lack of control on who physically completes the questionnaires, a challenge with an online survey, could also affect the results. Our survey was not representative of all areas across Canada. As commonly happens with surveys, recall bias may have influenced responses, particularly as a number of patients were diag- 
Moretto et al.

nosed with cancer over 3 years ago. ${ }^{13}$ Finally, the number of respondent urologists was relatively small.

\section{Conclusion}

Kidney cancer patients and caregivers would like to have more information about their cancer, long-term followup and potential complications. Despite the discrepancy between urologists and patients/caregivers regarding the recall of information provided, both groups agree that a SCP tailored to KC may be an effective measure to address this need. However, further research is required to determine the impact of this intervention on the long-term outcomes and patients/caregivers satisfaction. The Kidney Cancer Research Network of Canada Survivorship Initiative is currently developing a national SCP for KC survivors and will assess its implementation and impact on patient care.

Acknowledgements: Kidney Cancer Canada.

Competing interests: Dr. Moretto, J. Basiuk, and D. Maskens declare no competing financial or personal interests. Dr. Jewett is a member of the Advisory Board for Pfizer. He has also received grants from Novartis, GSK and Pfizer. Lastly, he has participated in clinical trials with Novartis, GSK and Pfizer. Dr. Canil has participated in Advisory Boards for Janssen, Pfizer, Astellas and Amgen. She has received speaking fees for Janseen and Astellas and travel grants from Novartis and Janssen. She has been an investigator in clinical trials for Pfizer, Janssen, Astellas and Sanofi Oncology.

This paper has been peer-reviewed.

\section{References}

1. Pantuck AJ, Zisman A, Belldegrun AS. The changing natural history of renal cell carcinoma. J Urol 2001;166:1611-23. http://dx.doi.org/10.1016/S0022-5347(05)65640-6

2. Harshman $\mathrm{LC}$, Xie W, Biarnason $\mathrm{GA}$, et al. Conditional survival of patients with metastatic renal-cell carcinoma treated with VEGF-targeted therapy: A population-based study. Lancet Oncol 2012;13:927-35. http://dx.doi.org/10.1016/S1470-2045(12)70285-1

3. Siegel R, DeSantis C, Virgo K, et al. Cancer treatment and survivorship statistics, 2012. CA Cancer I Clin 2012;62:220-41. http://dx.doi.org/10.3322/caac.21149

4. National Cancer Institute. About Cancer Survivorship Research: Survivorship Definitions. http://cancercontrol.cancer.gov/ocs/definitions.html. Accessed May 25, 2014.

5. From Cancer Patient to Cancer Survivor: Lost in Transition. Hewitt M, Greenfield S, Stovall E, editors: The National Academies Press; 2005

6. Investment in Research on Survivorship and Palliative and End of Life Care, 2005-2008. A Special Report fromThe Canadian Cancer Research Alliance's Survey of Government and Voluntary Sector Investment in Cancer Research. Toronto: CCRA. Canadian Cancer Research Alliance; 2011. hittp://www.ccra-acrc. ca. Accessed May 25, 2014.

7. TNM Classification of Malignant tumors. 7th ed. Sobin LH, Gospodarowicz MK, Wittekind C, editors. Oxford, UK: Wiley-Blackwell; 2009.

8. Rechis R, Arvey $S R$, Beckjord EB. Perspectives of a lifelong cancer survivor-improving survivorship care. Nat Rev Clin Oncol 2013;10:117-20. http://dx.doi.org/10.1038/nrclinonc.2012.212

9. Ligibel JA, Denlinger CS. New NCCN guidelines for survivorship care. J Natt Compr Canc Netw 2013;11(5 Suppl):640-4

10. Earle CC, Ganz PA. Cancer survivorship care: Don't let the perfect be the enemy of the good. J Clin Oncol 2012;30:3764-8. http://dx.doi.org/10.1200/JC0.2012.41.7667

11. Kassouf $W$, Siemens R, Morash C, et al. Follow-up guidelines after radical or partial nephrectomy for localized and locally advanced renal cell carcinoma. Can Urol Assoc J 2009;3:73-6.

12. ASCO Connection. ASCO Issues Recommendations for Improving Cancer Survivor Care in the United States 2013. http://connection.asco.org/Magazine/Article/ID/3418/ASCO-Issues-Recommendationsfor-Improving-Cancer-Survivor-Care-in-the-United-States.aspx. Accessed May 25, 2014.

13. Eisenhower D, Mathiowetz NA, Morganstein D. Recall Error: Sources and Bias Reduction Techniques. Measurement Errors in Surveys. John Wiley \& Sons, Inc.; 2004:125-44.

Correspondence: Dr. Christina M. Canil, Division of Medical Oncology, Department of Medicine, The Ottawa Hospital Cancer Centre, 501 Smyth Rd, Ottawa ON, Canada K1H 8L6; ccanil@ottawahospital.on.ca 